\title{
Review of: "The fliR gene contributes to the virulence of S. marcescens in a Drosophila intestinal infection model"
}

\author{
Ilan Rosenshein ${ }^{1}$ \\ 1 Hebrew University of Jerusalem
}

Potential competing interests: The author(s) declared that no potential competing interests exist.

A screen of Tn5 mutant library identified FliR as protein required for virulence of S. marcescens in the Drosophila intestinal infection model. FliR is a component of the flagella type III secretion system (f-T3SS). A comparison of fliR mutant to a mutant in the flagella master regulator, flhD, showed that both genes are required for translocation of the bacteria from the gut lumen into the hemocoel, indicating that the flagellum is required for the passage of bacteria through the intestinal barrier. In contrast, fliR but not flhD was convincingly shown to be needed to severely damage the intestinal epithelium and ultimately kill the host. The authors therefore suggest a flagellum-independent role for fliR in S. marcescens virulence in infected fly. This notion is novel and very interesting. How fliR or the flagella function to enhance virulence remain elusive.

\section{Comments}

1. In E. coli and Salmonella, the expression of fliR is flhD-dependent. The suggestion that FliR functions as a virulence factor in an flhD-independent manner implies that in S. marcescens fliR is expressed by an additional, alternative, FlhD-independent manner and this possibility can be tested.

1. How the flagella, FlhD, or FliR, function to promote virulence remain vague and this is the major weakness of this work. The authors raised the possibility that the flagella are needed for motility or for motility-independent processes such as protein secretion. Yet, these possibilities were not experimentally tested. Additional possibility that might account for the role of the flagella basal body in virulence is secretion of the anti-sigma $\mathrm{F}$ that might lead in turn to expression of some sigma $\mathrm{F}$ dependent non-flagella genes encoding virulence factor(s). An additional fascinating possibility is related to the FliR being a key component of the f-T3SS export apparatuses, shown to form an alternative non-flagella projection termed nanotube, which function for obtaining nutrient by pathogens from infected eukaryotic cells (PMID: 30929902, 30929979). It will be thus interesting to test the virulence of non flagellated mutants in genes required for nanotube formation (i.e., flip, fliQ, flhB, flhA, fliO) and mutants in genes required for flagella formation but not for nanotube formation (e.g., flil, FlgH, FliC). 
